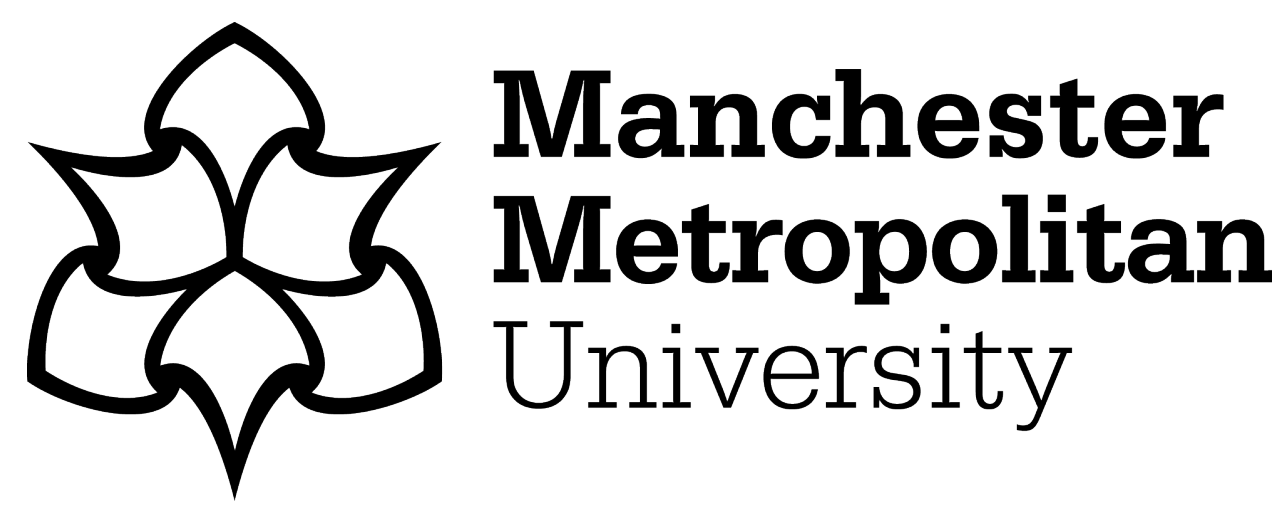

King, Laurie A ORCID logoORCID: https://orcid.org/0000-0002-0772-2378, Hubert, McKenzie A, Capuano, Christopher, Manco, Judith, Danilovic, Nemanja, Valle, Eduardo, Hellstern, Thomas R, Ayers, Katherine and Jaramillo, Thomas $F$ (2019) A non-precious metal hydrogen catalyst in a commercial polymer electrolyte membrane electrolyser. Nature Nanotechnology, 14 (11). pp. 1071-1074. ISSN 1748-3387

Downloaded from: https://e-space.mmu.ac.uk/624258/

Publisher: Nature Research

DOI: https://doi.org/10.1038/s41565-019-0550-7

Please cite the published version 


\section{Non-precious metal hydrogen catalyst in commercial polymer electrolyte membrane electrolysers}

Authors:

Laurie A. King, ${ }^{1 ¥}$ McKenzie A. Hubert, ${ }^{1 ¥}$ Christopher Capuano², Judith Manco, ${ }^{2}$ Nemanja Danilovic, ${ }^{3}$ Eduardo Valle, ${ }^{1}$ Thomas R. Hellstern ${ }^{1}$, Katherine Ayers, ${ }^{2}$ and Thomas F. Jaramillo*1

\section{Affiliations:}

${ }^{1}$ Department of Chemical Engineering, Shriram Center, Stanford University, 443 Via Ortega, Stanford, CA 94305, USA

${ }^{2}$ Nel Hydrogen/Proton OnSite, 10 Technology Drive, Wallingford, CT 06492, USA

${ }^{3}$ Lawrence Berkeley National Lab, 1 Cyclotron Road, Berkeley, CA 94720, USA

*Corresponding author: jaramillo@stanford.edu

\section{Abstract:}

We demonstrate the translation of a low cost, non-precious metal cobalt phosphide (CoP) catalyst from $1 \mathrm{~cm}^{2}$ lab-scale experiments to a commercial-scale $86 \mathrm{~cm}^{2}$ polymer electrolyte membrane (PEM) electrolyser. A 2-step bulk synthesis was adopted to produce CoP on a high surface area carbon support that was readily integrated into an industrial PEM electrolyser fabrication process. The performance of the CoP was compared head-to-head with a platinum-based PEM under the same operating conditions ( $400 \mathrm{psi}, 50^{\circ} \mathrm{C}$ ). CoP was found to be active and stable, operating at $1.86{\mathrm{~A} . \mathrm{cm}^{-2}}^{-2}$ for $>1700$ hours of continuous hydrogen production while providing substantial material cost savings relative to platinum. This work illustrates a potential pathway for non-precious hydrogen evolution catalysts developed in past decades to translate to commercial applications. 


\section{Main Text:}

The modern-day commercialisation of large-scale polymer electrolyte membrane (PEM) water electrolysers represents the culmination of decades of fundamental scientific studies involving catalysts, membranes, electrode architectures, and membrane electrode assemblies (MEAs) among other key advancements. Commercial PEM electrolysers utilize expensive platinum group catalysts such as platinum and iridium, despite their high costs and scarcity, to achieve and maintain high activity over 50,000 h. Terawatt-scale deployment of electrolysers envisioned for grid resilience require significant reduction of platinum group catalysts and/or replacement by alternative nonplatinum group (NPG) catalysts within the device (1). This goal presents many challenges, including the need to tailor catalyst inks, tune deposition methods, and provide stable activity at elevated temperatures, pressures, and current densities, all while demonstrating high activity and durability over the lifetime expected of a commercial system. In this study, we bridge the gap between decades of lab-scale, solution-based, NPG catalyst development and industrial-scale electrolyser operation. We report the first integration of a highly active NPG catalyst (cobalt phosphide, CoP) into a commercial grade PEM electrolyser with $>1700$ hours of continuous operation and negligible loss in activity. We briefly discuss the economic trade-off of replacing the traditional Pt cathode with a NPG catalyst while emphasizing the promise of NPG materials to supersede precious metal catalysts in commercial PEM electrolysers.

Recent technoeconomic analyses suggest that PEM electrolysis is a promising technology for widespread renewable hydrogen production, hinging on capital cost reductions that would make this technology competitive in the hydrogen market (1). Overcoming this challenge requires cost reductions across many system components, demanding substantial innovation in material design, engineering, and manufacturing. Reducing precious metal content has been a major R\&D effort among manufacturers of PEM electrolysers, as doing so would reduce capital costs while also mitigating issues of material scarcity. While Pt and Ir catalysts constitute $\sim 8 \%$ of the total stack cost today, price inelasticity, and therefore volatility, of these precious metals hinder future TWscale deployment of PEM electrolysis (2). This motivates the development of active and stable NPG hydrogen evolution reaction (HER) catalysts, the best-case scenario for reducing Pt content in PEM electrolysers. 
Inspired by hydrogen-producing enzymes such as nitrogenase, molybdenum disulphide $\left(\mathrm{MoS}_{2}\right)$ emerged as one of the first promising NPG HER catalysts $(3,4)$. Subsequently, researchers pursued similar classes of materials, including transition metal phosphides, which exhibit favourable activities and stabilities in lab scale demonstrations (5-7). Significant advances in the activities of NPG catalysts have been accomplished by tuning the morphology, chemical composition, and crystal structure via synthesis methods (5, 6). Universal to nearly all published NPG HER literature are the electrochemical testing protocols used to measure catalyst activity and stability (linear sweep voltammetry, cyclic voltammetry, chronoamperometry, and/or chronopotentiometry) (8). Such methodologies provide figures of merits such as onset potential, exchange current densities, and Tafel slopes which enable benchmarking, as well as provide insightful fundamental knowledge. However, relatively few studies have translated NPG catalysts to PEM electrolyser devices (9-12) and to the best of our knowledge, there are no reports demonstrating their integration in commercial-scale systems at relevant sizes, current densities, pressures, and temperatures.

Herein, we report the first translation of a NPG HER catalyst into a commercial-scale PEM electrolyser. Thanks to the development efforts of many, there were a multitude of potential NPG catalyst candidates $(5,13,14)$. For this first demonstration we selected CoP, as experimental and theoretical studies have shown it to have promising HER activity in the acidic environments relevant for PEM electrolysers (15-19). The CoP was prepared by wetness impregnation of cobalt nitrate onto Vulcan carbon followed by vapour phase phosphidation. The phosphidation step was adapted from a previous report wherein a $\mathrm{CoP}$ thin film was fabricated on a silicon substrate (18). The direct growth of the phosphide on the carbon support facilitates mechanical adhesion of the catalyst to the substrate and enhances uniform electrical conductivity and dispersion of the catalyst (6). Our simple 2-step synthesis produced $>1.1$ gram powder catalyst batch sizes, amenable to MEA fabrication using ink-based methods. This method has the potential for orders of magnitude scale-up, as batch size was only limited by the reactor size employed. Synthetic details are provided in the supplementary information.

Shown in Fig. 1A is the X-ray diffraction (XRD) pattern of the as-synthesised catalyst, confirming that our developed method generates a phase-pure catalyst (FeAs-type CoP). 
X-ray photoelectron spectroscopy (XPS) of the as-prepared catalyst shows the presence of CoP, cobalt oxide, and phosphate species (Fig. S1), which are commonly reported for CoP (20). The loading of CoP on the Vulcan carbon support was $\sim 21 \mathrm{wt} \%$ with a Co:P molar ratio of 1:1, determined using inductively coupled plasma mass spectrometry (ICPMS) (Tables S1 and S2).
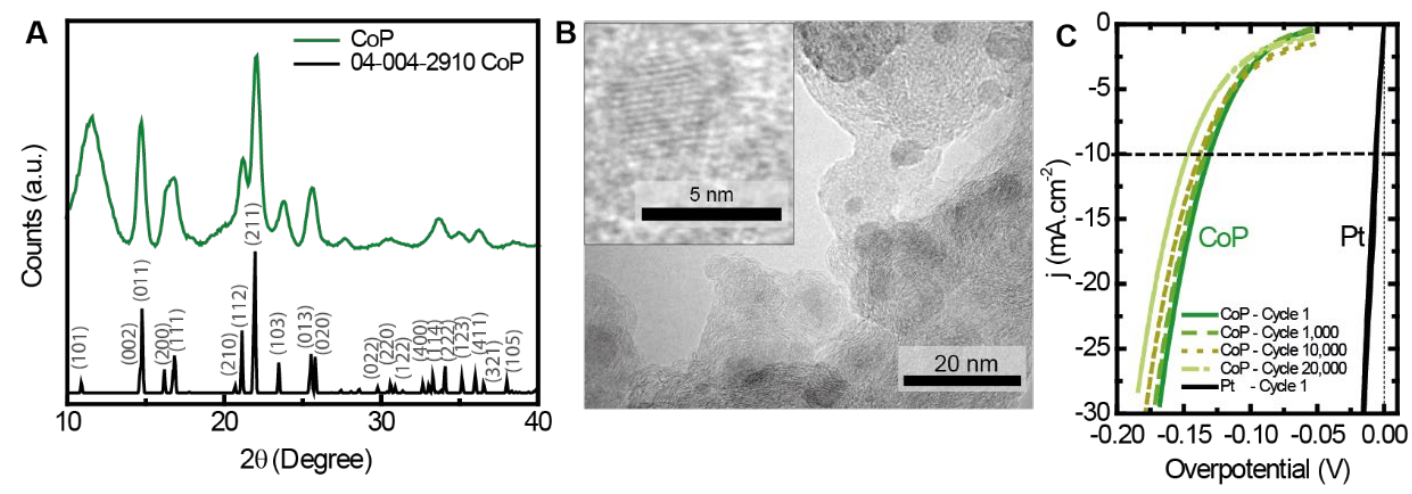

Fig. 1. Physical and electrochemical characterisation of the CoP catalyst. (A) Powder XRD. (B) TEM micrograph. Inset shows a higher magnification micrograph of a CoP nanoparticle. (C) Linear sweep voltammograms (LSVs) (iR-corrected) of the CoP catalyst $\left(0.12 \mathrm{mgCoP.cm}^{-2}\right)$ and commercial Pt catalyst $\left(0.12 \mathrm{mgPt}_{\mathrm{cm}}{ }^{-2}\right)$ drop cast onto carbon paper and tested in a 3-electrode electrochemical cell in $0.5 \mathrm{M} \mathrm{H}_{2} \mathrm{SO}_{4}$. LSVs are shown for the as-prepared CoP and commercial Pt (cycle 1), as well as after 1,000, 10,000 and 20,000 cycles of accelerated cyclic voltammograms for the CoP catalyst.

The morphology of the catalyst was examined with transmission electron microscopy (TEM) and scanning electron microscopy (SEM) (Fig. 1B and Fig. S2). TEM enables clear identification of CoP nanoparticles with an average diameter of $\sim 5 \mathrm{~nm}$ (Fig. 1B). HR-TEM (Fig. 1B inset) confirms lattice plane spacing of 2.5 Å which matches FeAs-type CoP (111) (16). Lower magnification TEM micrographs show that $\mathrm{CoP}$ is well-dispersed on the Vulcan carbon support (Fig. S3). Thus, we report a facile synthesis route, consisting of simple precursors and few processing steps, which yields a high surface area nanoparticulate CoP catalyst that is amenable to scaled-up MEA fabrication requiring minimal technical changes.

Lab-scale measurements, in acidic electrolyte (Fig. 1C) revealed excellent activity and stability of the CoP catalyst (Fig. S4 and Fig. S5), in-line with previous studies $(21,22)$. For comparison, the activity of a commercial Pt catalyst of similar loading is also shown (Fig. 1C). While the activity of the CoP catalyst does not rival that of Pt, CoP showed little 
degradation under lab-scale cyclic voltammetry durability testing conditions and reasonable onset potentials. It is well known that 3-electrode stability measurements do not necessarily translate into MEA stability (23-25), which motivated further examination of the CoP catalyst in a MEA. Although CoP is not the most active catalyst in the literature (5), it represents a platform for performing these initial and important feasibility screenings of NPG catalysts within a commercial-grade electrolyser, motivating exploration of other NPG catalysts within these systems.

To fabricate cathode gas diffusion electrodes (GDEs), the catalyst was dispersed in a suspension of Nafion ionomer, IPA, and water, then spray coated onto carbon paper gas diffusion layers. The anode GDE used $\mathrm{IrO}_{\mathrm{x}}$ as the baseline material, a known durable catalyst, which isolated any observed performance changes in the electrolyser to the NPG cathode. A Nafion 117 proton exchange membrane was used in the MEA, placed between the anode and cathode GDEs. The MEAs were assembled in Proton OnSite's commercial $86 \mathrm{~cm}^{2}$ active area PEM-electrolyser cell stack (Fig. 2A) along with a baseline all-PGM cell (Pt black-based GDE) for direct comparison under identical operating conditions. All tests were conducted at 400 psi hydrogen differential pressure and at elevated temperatures with liquid water fed to the anode side of the cell. Table S3 highlights the distinct differences between the 3-electrode lab-scale setup and the commercial-grade testing protocol.

The CoP catalyst was loaded at $1.0 \mathrm{mg}_{\mathrm{Cop} . \mathrm{cm}^{-2}}$ on the GDE, equivalent to $4.5 \mathrm{mg}^{\mathrm{cm}}{ }^{-2}$ total catalyst loading including the carbon support. The electrolyser was first characterised by stepping the current from $100 \mathrm{~mA} \cdot \mathrm{cm}^{-2}(1.54 \mathrm{~V})$ to $1.86{\mathrm{~A} . \mathrm{cm}^{-2}}^{2}\left(2.27 \mathrm{~V}\right.$ ) at $50{ }^{\circ} \mathrm{C}$ (Fig. 2B). Interestingly, the polarisation curve shows this non-precious catalyst can reach the same high current densities as the Pt-containing cell at reasonable potentials. Assuming that every unit of CoP is active for the HER, a conservative estimate for the turnover frequency (TOF) of CoP is $0.87 \mathrm{H}_{2} . \mathrm{s}^{-1}$. More likely, only the surface bound CoP units ( 10 $\%$ ) participate in the reaction, leading to an estimated TOFavg of $8.7 \mathrm{H}_{2} \cdot \mathrm{s}^{-1}$. Comparatively, the TOF for the Pt catalyst is estimated to be $1.88 \mathrm{H}_{2} \cdot \mathrm{s}^{-1}$ conservatively, or $28.8 \mathrm{H}_{2} \cdot \mathrm{s}^{-1}$ assuming only surface atoms participate in the reaction. Details of the calculations are shown in the supplementary information.

The all-PGM baseline GDE shows that the Pt PEM required 2.05 V to reach a current density of $1.86{\mathrm{~A} . \mathrm{cm}^{-2}}^{-2}$ (Fig. 2B). This result reflects operating efficiencies of $55 \%$ and $61 \%$ 
respectively for the CoP- and Pt-based electrolysers based on the lower heating value (LHV). While the fact that the Pt system performs $220 \mathrm{mV}$ better than the NPG catalyst is not surprising given the 3-electrode measurements (Fig. 1C), this small difference is impressive considering the commercially relevant high current densities employed. Future work will explore electrode engineering to maximize the performance of NPG based electrolysers. Optimization of catalyst loading and ionomer content, for example, will only help to narrow the performance gap between PGM and NPG catalysts in commercial electrolyser platforms. This gap will continue to shrink as NPG catalysts themselves are further improved, minimizing the trade-offs between operating and capital costs (14).
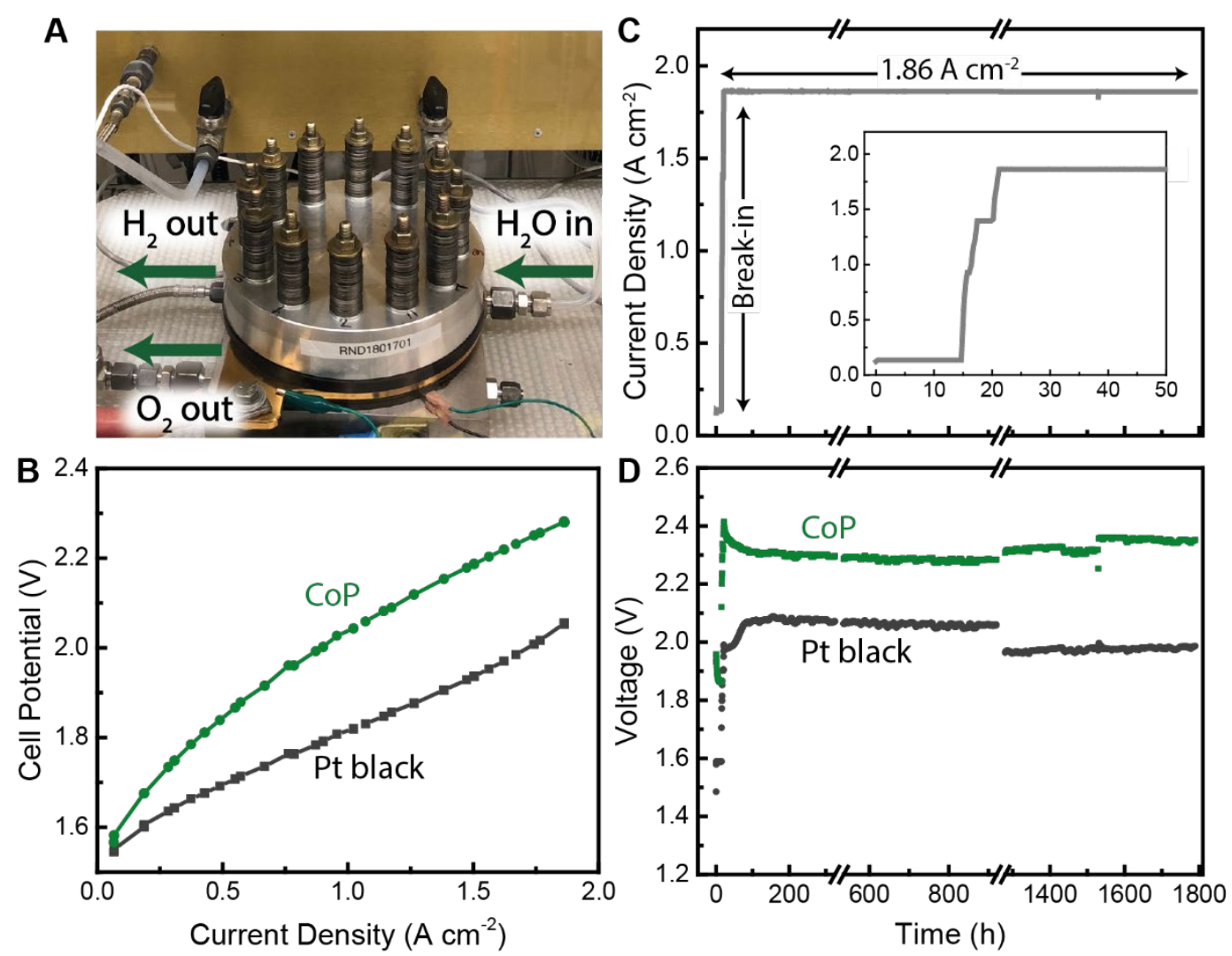

Fig. 2. PEM electrolyser performance. (A) Photograph of the $86 \mathrm{~cm}^{2}$ electrolyser test station. (B) Polarisation curve. (C) Current density profile for the durability protocol. The inset shows the current density profile for the break-in period only. (D) Durability voltage-time plots for the PEM electrolyser prepared with $1.0 \mathrm{mg}_{\mathrm{Cop} . \mathrm{cm}^{-2}}$ loading while operating at $50^{\circ} \mathrm{C}$ and $400 \mathrm{psi}$. Voltage discontinuities result from power outage-induced restarts. 
The CoP and Pt-based MEA durability tests were also conducted in the 2-cell stack following the polarisation studies. The differences in operating conditions between the 2-electrode and 3-electrode stability measurements are noted in Table S3. The tests were

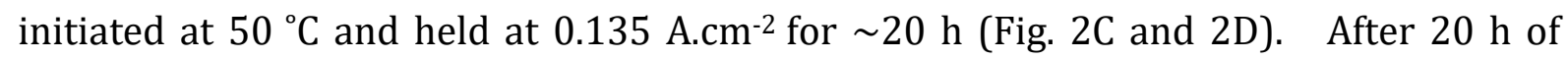
operation, the temperature was ramped to $55^{\circ} \mathrm{C}$ and the current was increased to 1.86

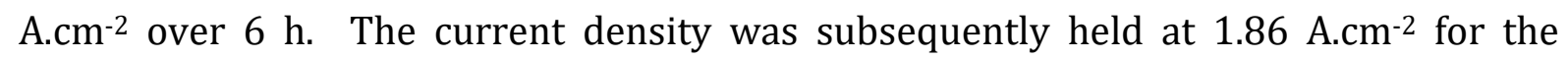
remaining $1763 \mathrm{~h}$ of testing. During the durability measurement, the CoP MEA stabilised within $50 \mathrm{~h}$ to provide steady operation at $2.30 \mathrm{~V}$ for $>900 \mathrm{~h}$ with energy consumption of $60.4 \mathrm{kWh} . \mathrm{kgH}^{-1}$. The Pt-based MEA operated under the same conditions $\left(1.86{\mathrm{~A} . \mathrm{cm}^{-2}}^{2}, 55\right.$ ${ }^{\circ} \mathrm{C}, 400 \mathrm{psi}$ ) at $2.06 \mathrm{~V}$ as expected without degradation, corresponding to energy consumption of $54.5 \mathrm{kWh} . \mathrm{kg}_{\mathrm{H}}{ }^{-1}$. Transient behaviour in the break-in phase is discussed in the supplementary information.

After $922 \mathrm{~h}$ of continuous testing, the 2-cell stack (CoP and Pt MEAs) lost power briefly $(<1 \mathrm{~min})$ and was restarted. The reset resulted in an increase $(\sim 50 \mathrm{mV})$ in cell potential for the CoP MEA and a slightly reduced potential for the Pt MEA $(\sim 6 \mathrm{mV})$. A second, small abrupt increase in potential was observed after $1550 \mathrm{~h}$ of operation, likely due to a second disruption in the applied potential. Duplicate CoP and Pt MEAs were fabricated and tested with a similar protocol and were shown to be extremely stable with comparable cell potentials (Fig. S6). Discussion of the MEA restarts and characterisation of the CoP GDE after the $1700 \mathrm{~h}$ durability test are provided in the supplementary information (Fig. S7). Analogous to the fuel cell literature, it is important to note that Pt-based commercial PEM electrolysers are also known to degrade on start-up and shut-down cycling and hence this challenge is relevant for all known catalysts, precious metal and non-PGMs alike (26).

In a 3-electrode lab-scale configuration, it has been reported that non-precious metal ionic compound catalysts such as transition metal phosphides dissolve at higher rates under OCP conditions, but are stable when evolving hydrogen, even when cycling from low to high production rates $(27,28)$. This report is consistent with our lab-scale durability measurements of CoP dissolution during electrochemical testing (Fig. S8 and Fig. S9). We therefore postulate that CoP dissolution occurred when the MEA lost power briefly after $922 \mathrm{~h}$ and $1550 \mathrm{~h}$ of operation leading to the increased cell potential. 
Furthermore, we propose operating procedures that maintain a small operating current could prevent this degradation mechanism.

Remarkably, the CoP MEA produced a total of $10.1 \mathrm{~kg}$ of $\mathrm{H}_{2}$ (912 L at STP), and we conservatively estimate a turnover number (TON) of $5.3 \times 10^{6} \mathrm{H} 2$ molecules produced per CoP site assuming all CoP units are active. Details of the calculations are shown in the supplementary information. The CoP MEA requires 12 - $18 \%$ greater power density than the Pt baseline MEA, a potentially attractive trade-off between electricity consumption and electrolyser capital cost depending on the application and the local cost of electricity. While this work highlights a significant step towards non-PGM catalyst incorporation in commercial electrolysers, the difference in performance between CoP and $\mathrm{Pt}$ is still too substantial for wide-scale commercial deployment, motivating continued research and development of non-PGM catalysts.

Analyses show that the levelized cost of hydrogen (LCH) from water electrolysis is currently dominated by the cost of electricity $(29,30)$. However, as society follows a path towards increased electrification and electricity prices drop, the capital cost of electrolysers will begin to dominate $(31,32)$. Utilisation of durable, low-cost NPG catalysts that can replace expensive and price inelastic precious metals is one critical step towards reducing the overall capital cost of PEM electrolysis (Table S4). While the result presented here is a significant achievement, we note that cost reductions across all system components are needed for TW-scale deployment of PEM electrolysers. A full techno-economic analysis on the implications of this report is outside the scope and will be the subject of future investigations.

Our initial demonstration of an active and highly stable NPG HER catalyst in a commercial-scale PEM electrolyser highlights the practical relevance of NPG systems. By extending our lab-scale stability study from liquid electrolyte and $1 \mathrm{~cm}^{2}$ electrode area to an $86 \mathrm{~cm}^{2}$ commercial PEM electrolyser with $>1700 \mathrm{~h}$ of stable operation at elevated temperature and pressure, we demonstrate the commercial relevance of NPG catalysts. Compared to a Pt-based PEM, we found the CoP PEM to pay a 12 - $18 \%$ power penalty under the operating conditions, but to provide a significant improvement in material cost over the commercial Pt catalyst. We believe that the results represent a possible entry point for NPG catalyst utilisation in commercial water electrolysers. Continued research efforts between industry and academia enables pathways to lowering the capital 
investment costs of PEM electrolysers while maintaining high operating efficiencies to implement grid-scale energy storage.

\section{Methods}

Methods, including statements of data availability are available in the Supplementary Information.

\section{References and Notes}

1. L. Bertuccioli et al., Study on development of water electrolysis in the EU. Fuel Cells Hydrog. Jt. Undert., 1-160 (2014).

2. P. C. K. Vesborg, T. F. Jaramillo, Addressing the terawatt challenge: scalability in the supply of chemical elements for renewable energy. RSC Adv. 2, 7933 (2012).

3. B. Hinnemann et al., Biomimetic Hydrogen Evolution: MoS2 Nanoparticles as Catalyst for Hydrogen Evolution. J Am Chem Soc. 36, 5308-5309 (2005).

4. T. F. Jaramillo et al., Identification of Active Edge Sites for Electrochemical H2 Evolution from MoS2 Nanocatalysts. Science (80-. ). 317, 100-102 (2007).

5. J. F. Callejas, C. G. Read, C. W. Roske, N. S. Lewis, R. E. Schaak, Synthesis, Characterization , and Properties of Metal Phosphide Catalysts for the Hydrogen-Evolution Reaction. Chem. Mater. (2016), doi:10.1021/acs.chemmater.6b02148.

6. Y. Shi, B. Zhang, Recent advances in transition metal phosphide nanomaterials: synthesis and applications in hydrogen evolution reaction. Chem. Soc. Rev. 45, 1529-1541 (2016).

7. J. Kibsgaard, T. F. Jaramillo, Molybdenum phosphosulfide: An active, acid-stable, earthAbundant catalyst for the hydrogen evolution reaction. Angew. Chemie - Int. Ed. 53, 14433-14437 (2014).

8. C. C. L. McCrory et al., Benchmarking Hydrogen Evolving Reaction and Oxygen Evolving Reaction Electrocatalysts for Solar Water Splitting Devices. J. Am. Chem. Soc. 137, 43474357 (2015).

9. T. Corrales-Sánchez, J. Ampurdanés, A. Urakawa, MoS2-based materials as alternative cathode catalyst for PEM electrolysis. Int. J. Hydrogen Energy. 39, 20837-20843 (2014).

10. J. W. D. Ng et al., Polymer Electrolyte Membrane Electrolyzers Utilizing Non-precious Mobased Hydrogen Evolution Catalysts. ChemSusChem. 8, 3512-3519 (2015).

11. C. Di Giovanni et al., Low-Cost Nanostructured Iron Sulfide Electrocatalysts for PEM Water Electrolysis. ACS Catal. 6, 2626-2631 (2016).

12. X. Sun et al., Earth-Abundant Electrocatalysts in Proton Exchange Membrane Electrolyzers. Catalysts. 8, 657 (2018).

13. S. Anantharaj et al., Recent Trends and Perspectives in Electrochemical Water Splitting with an Emphasis on Sulfide, Selenide, and Phosphide Catalysts of Fe, Co, and Ni: A Review. ACS Catal. 6, 8069-8097 (2016).

14. P. Xiao, W. Chen, X. Wang, A Review of Phosphide-Based Materials for Electrocatalytic 
Hydrogen Evolution. Adv. Energy Mater. 5, 1-13 (2015).

15. W. Liu et al., A highly active and stable hydrogen evolution catalyst based on pyritestructured cobalt phosphosulfide. Nat. Commun. 7, 1-9 (2016).

16. E. J. Popczun, C. G. Read, C. W. Roske, N. S. Lewis, R. E. Schaak, Highly Active Electrocatalysis of the Hydrogen Evolution Reaction by Cobalt Phosphide Nanoparticles ** Angewandte. Angew. Chemie - Int. Ed., 5531-5534 (2014).

17. J. Kibsgaard et al., Designing an improved transition metal phosphide catalyst for hydrogen evolution using experimental and theoretical trends. Energy Environ. Sci. 8, 3022-3029 (2015).

18. T. R. Hellstern, J. D. Benck, J. Kibsgaard, C. Hahn, T. F. Jaramillo, Engineering Cobalt Phosphide ( CoP ) Thin Film Catalysts for Enhanced Hydrogen Evolution Activity on Silicon Photocathodes. Adv. Energy Mater. (2016), doi:10.1002/aenm.201501758.

19. Z. Wu, L. Huang, H. Liu, H. Wang, Element-Specific Restructuring of Anion- and CationSubstituted Cobalt Phosphide Nanoparticles under Electrochemical Water-Splitting Conditions. ACS Catal. 9, 2956-2961 (2019).

20. F. H. Saadi et al., Operando Spectroscopic Analysis of CoP Films Electrocatalyzing the Hydrogen-Evolution Reaction. J. Am. Chem. Soc. 139, 12927-12930 (2017).

21. H. Yang, Y. Zhang, F. Hu, Q. Wang, Urchin-like CoP Nanocrystals as Hydrogen Evolution Reaction and Oxygen Reduction Reaction Dual-Electrocatalyst with Superior Stability. Nano Lett. 15, 7616-7620 (2015).

22. D. Zhou et al., Interconnected urchin-like cobalt phosphide microspheres film for highly efficient electrochemical hydrogen evolution in both acidic and basic media. J. Mater. Chem. A. 4, 10114-10117 (2016).

23. S. Martens et al., A comparison of rotating disc electrode, floating electrode technique and membrane electrode assembly measurements for catalyst testing. J. Power Sources. 392, 274-284 (2018).

24. S. M. Alia et al., Activity and Durability of Iridium Nanoparticles in the Oxygen Evolution Reaction. J. Electrochem. Soc. 163, F3105-F3112 (2016).

25. S. M. Alia, B. S. Pivovar, Iridium-Based Nanowires as Highly Active, Oxygen Evolution Reaction Electrocatalysts. ACS Catal. 8, 2111-2120 (2018).

26. A. Weiß, A. Siebel, M. Bernt, H. A. Gasteiger, Impact of Intermittent Operation on the Lifetime and Performance of a PEM Water Electrolyzer. Meet. Abstr. . MA2018-02, 1606 (2018).

27. Y. Zhang, L. Gao, E. J. M. Hensen, J. P. Hofmann, Evaluating the Stability of Co $2 \mathrm{P}$ Electrocatalysts in the Hydrogen Evolution Reaction for Both Acidic and Alkaline Electrolytes. ACS Energy Lett., 1360-1365 (2018).

28. M. Ledendecker et al., Stability and Activity of Non-Noble-Metal-Based Catalysts Toward the Hydrogen Evolution Reaction. Angew. Chemie. 129, 9899-9903 (2017).

29. B. James, W. Colella, J. Moton, G. Saur, T. Ramsden, PEM Electrolysis H2A Production Case Study Documentation. PEM Electrolysis H2A Prod. Case Study Doc., 1-27 (2013).

30. U. Babic, M. Suermann, F. N. Büchi, L. Gubler, T. J. Schmidt, Critical Review-Identifying Critical Gaps for Polymer Electrolyte Water Electrolysis Development. J. Electrochem. Soc. 164, F387-F399 (2017). 
31. I. Introduction, Introduction - DOE 2013 Hydrogen and Fuel Cells Program Annual Progress Report (2017).

32. F. Cell, T. Office, N. Renewable, 2017 H2 @ Scale Workshop Report (2017).

\section{Acknowledgements:}

Physical characterisation of the catalyst in this work was performed at the Stanford Nano Shared Facilities (SNSF), supported by the National Science Foundation under award ECCS-1542152. We thank Richard Chin for his assistance with scanning electron microscopy at this facility.

Funding: We acknowledge DoD SBIR Phase I Contract Number: N00024-17-P-4507, topic Number: N162-107 for financial support and the project manager Joshua Manney. The US Department of Energy (DOE) Office of Basic Energy Sciences (BES) is gratefully acknowledged for support for the SUNCAT Center for Interface Science and Catalysis for fundamental catalysis development. M.A.H acknowledges the support of a National Science Foundation Graduate Research Fellowship.

Authors contributions: L.A.K. and M.A.H. contributed equally to this work. L.A.K., M.A.H., and T.R.H. synthesized CoP catalysts. L.A.K. performed x-ray diffraction and scanning electron microscopy characterisation. E.V. performed transmission electron microscopy characterisation. M.A.H. performed electrochemical lab-scale characterisation and testing. C.C., J.M., and N.D. prepared all PEM stack components, assembled and tested the electrolyser, as well as collected all operational data. L.A.K. and M.A.H. contributed to data analysis, including preparation and revision of this manuscript.

\section{Competing interests: None.}

Data and materials availability: The x-ray diffraction reference pattern is available from the Materials Project database under the ID mp-22270. Full synthetic details, electrochemical testing protocols, and raw data can be found in the supplementary information section. 


\section{Supplementary Information}

349 Materials and Methods

350 Figures S1 - S9

351 Table S1 - S4

352 Turnover frequency and turnover number estimations

353 Electrolyser efficiency calculations

354 